$$
\text { "antibi" — 2005/2/16 — 20:14 — page 319 — \#1 }
$$

\title{
Une modélisation d'un zoom au moyen de microscopes virtuels
}

\author{
André Antibi, Jacques Bair et Valérie Henry
}

Abstract. In this paper, we explain how a computer works when "zooms" are made around a point on a planar curve. This modelisation leads to an easy and algorithmic method to find the (vertical or not vertical) tangents for the studied curve.

Mots-clé: Zoom, microscope virtuel, tangente, point de non dérivabilité, limite de courbes, convergence uniforme, didactique des mathématiques.

ZDM Subject Classification: I10, P7, R20.

\section{Introduction}

Le concept de "zoom", bien connu dans la vie courante, peut être exploité en analyse mathématique dès qu'il est question d'étudier une notion locale.

Dans cet article, nous nous attachons à la représentation d'une courbe plane par un ordinateur (ou une calculatrice graphique) avec lequel sont réalisés des zooms successifs autour d'un même point appartenant à cette courbe.

Après deux exemples introductifs destinés à fournir une représentation visuelle de l'effet de zooms sur une courbe, nous modélisons ce phénomène en recourant à la notion de microscope virtuel, ce qui nous conduit à envisager des microscopes virtuels dont la "puissance" devient de plus en plus grande, puis inévitablement à nous interroger sur le résultat fourni par des microscopes virtuels dont la puissance tend vers l'infini.

Copyright (C) 2004 by University of Debrecen 
En nous basant sur la notion de limite d'une suite de courbes, nous montrons comment l'application de microscopes virtuels à une courbe représentative d'une fonction dérivable conduit à une équation de la tangente à la courbe au point autour duquel sont effectués les zooms successifs. Ceci nous permet ainsi d'unifier deux conceptions classiques de la notion de tangente à une courbe : il s'agit de la limite de sécantes, mais aussi de la limite de courbes obtenues par des zooms successifs.

Nous poursuivons en considérant le cas de fonctions non dérivables pour reconnaître, sur quelques exemples, l'existence d'un point anguleux, d'un point de rebroussement ou d'une tangente verticale.

Nous terminons par une section consacrée aux courbes définies implicitement et qui ne sont pas nécessairement représentatives d'une fonction à une variable réelle. Nous traitons en particulier le cas des coniques.
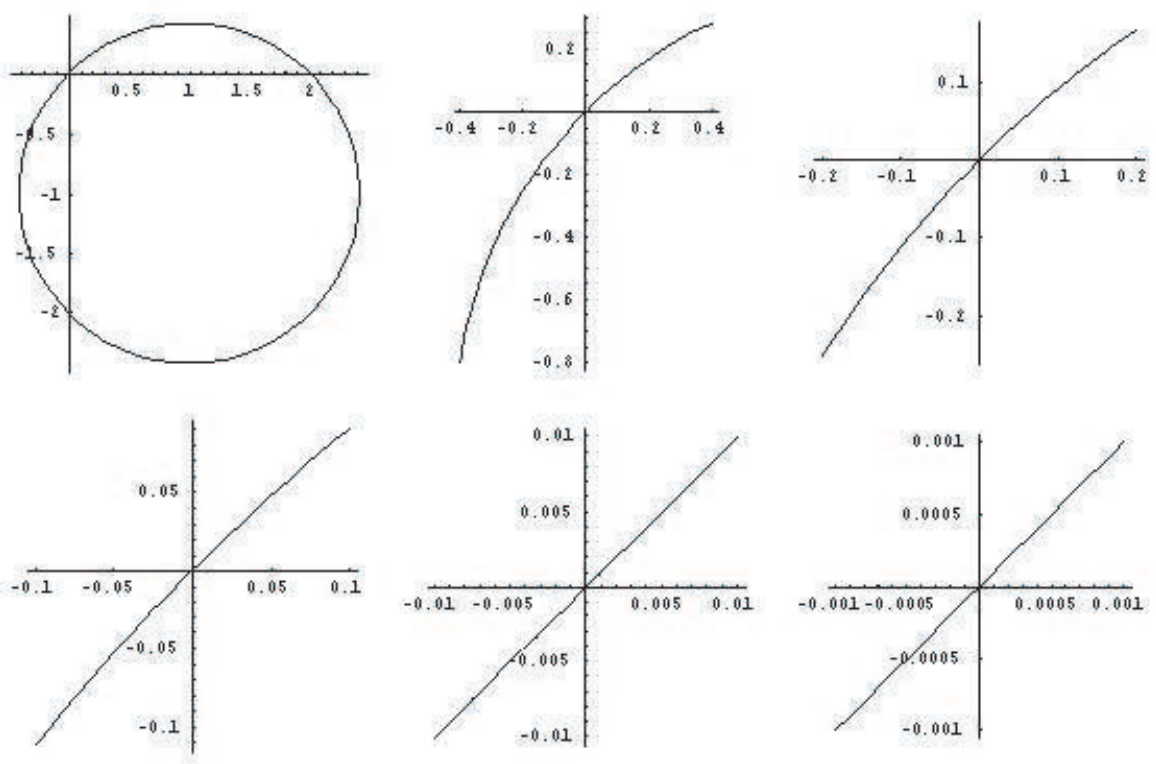

FIG. 1. Cercle d'équation $(x-1)^{2}+(y+1)^{2}=2$ au voisinage de l'origine

Signalons encore que notre texte est parsemé de réflexions d'ordre didactique et contient également diverses suggestions d'activités pédagogiques connexes à ces problèmes; elles peuvent être réalisées dans le cadre d'un enseignement de 
base en analyse mathématique, au niveau du Lycée ou d'une première année à l'université.

\section{Exemples introductifs}

Considérons le cercle centré sur le point $P(1 ;-1)$ et de rayon $\sqrt{2}$ (Figure 1 ), donc d'équation

$$
(x-1)^{2}+(y+1)^{2}=2
$$

il passe par l'origine $O(0 ; 0)$.

Lorsqu'un logiciel de calcul formel représente ce cercle, puis des portions de ce cercle pour des abscisses $x$ parcourant des intervalles symétriques autour de $O$ de plus en plus restreints, par exemple, sur les intervalles $[-0,4 ; 0,4],[-0,2 ; 0,2]$, $[-0,1 ; 0,1],[-0,01 ; 0,01]$ et $[-0,001 ; 0,001]$, nous obtenons les graphiques de la figure 1 .

Les résultats sont fort semblables quand nous effectuons des zooms successifs sur ce même cercle, mais cette fois autour du point $P(2 ; 0)$ (Figure 2).
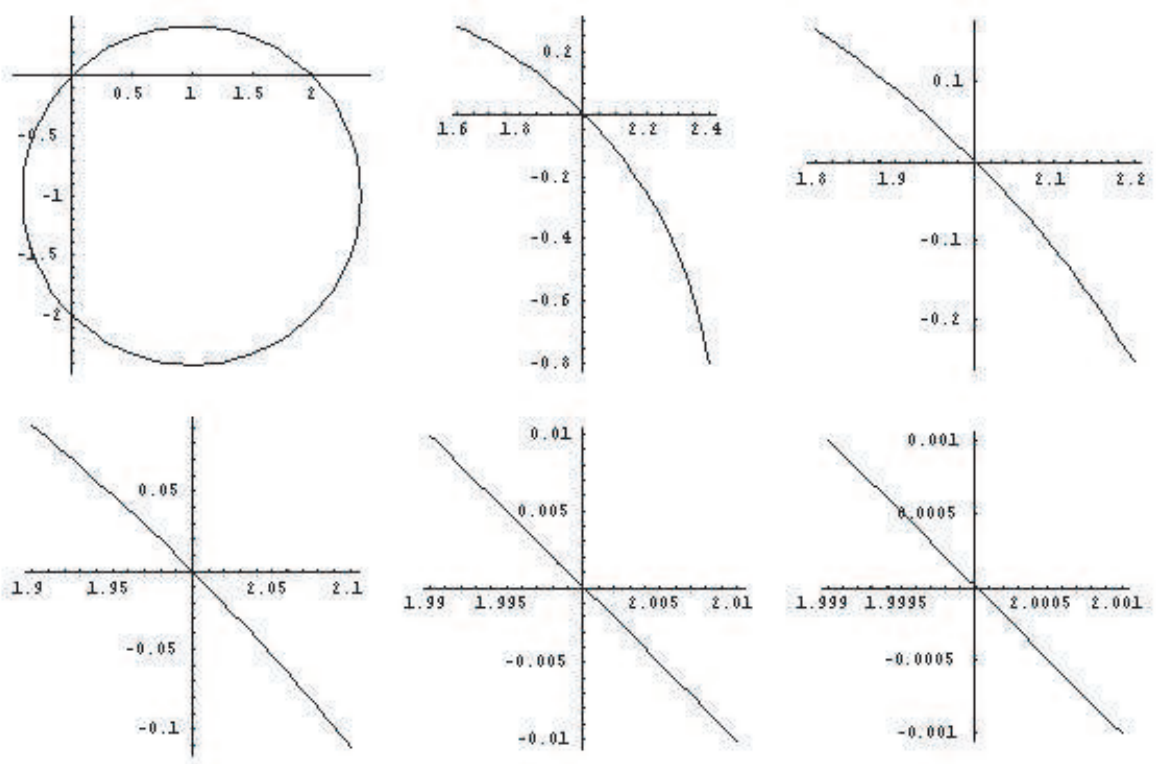

Fig. 2. Cercle d'équation $(x-1)^{2}+(y+1)^{2}=2$ au voisinage du point $(2 ; 0)$ 
Un examen de ces figures nous amène à constater deux phénomènes différents. D'une part, l'ordinateur nous fournit des images remplissant toujours tout l'éc$\operatorname{ran}^{1}$, même lorsque l'intervalle se rétrécit. D'autre part, la courbe semble "se rectifier" progressivement pour se présenter finalement sous la forme d'une droite qui ne sera plus modifiée par des zooms ultérieurs.

Nous nous proposons d'étudier ces deux phénomènes, tout d'abord en analysant le mode de fonctionnement de "microscopes virtuels".

\section{Notion de microscope virtuel}

Il est bien connu qu'un biologiste qui souhaite observer de minuscules microbes, par exemple, a recours à un microscope qui grossit les objets observés. De la même manière, un analyste qui cherche à étudier localement une courbe au voisinage d'un point peut "zoomer" sur la courbe en faisant appel à ce que nous allons appeler un microscope virtuel : il peut ainsi visualiser le graphique sur un intervalle très petit contenant l'abscisse considérée, son ordinateur se chargeant d'agrandir la zone observée.

L'exploitation de microscopes virtuels par des mathématiciens s'est largement répandue ces derniers temps avec l'avènement de l'analyse non-standard ([4], [7], [10], [12], [13], [15]). Elle n'est toutefois pas neuve, ainsi qu'en atteste cette réflexion de Pascal, qui donnait déjà en 1654 ce conseil pédagogique à ceux qui éprouvaient des difficultés à concevoir des quantités "infiniment petites" : s'ils ne peuvent comprendre que des parties si petites, qu'elles nous sont imperceptibles, puissent être autant divisées que le firmament, il n'y a pas de meilleur remède que de les leur faire regarder avec des lunettes qui grossissent cette pointe délicate jusqu'à une prodigieuse masse ([14], p. 30).

Afin de présenter notre modélisation d'un zoom, reprenons l'exemple introductif du cercle $\mathcal{C}$ d'équation

$$
(x-1)^{2}+(y+1)^{2}=2
$$

et observons, dans un premier temps, ce qui se passe au voisinage immédiat de l'origine. Pour bien cerner le comportement du cercle près de l'origine, nous devons demander à la machine de tracer la courbe sur un intervalle très réduit; mais, si la figure était représentée dans un référentiel "habituel", elle ne serait guère claire,

${ }^{1}$ Dans la pratique, il s'agit plus souvent d'une fenêtre d'écran. Dans la suite, nous utiliserons néanmoins toujours le terme "écran". 


$$
\text { "antibi" — 2005/2/16 — 20:14 — page 323 — \#5 }
$$

car des points trop proches de l'origine seraient indiscernables; par exemple, les points d'abscisses $-\frac{1}{10}, 0$ et $\frac{1}{10}$ sembleraient confondus. C'est pourquoi, un logiciel fournit automatiquement une image "agrandie".

Décrivons le fonctionnement du logiciel.

La machine dispose d'un écran, supposé carré, de dimensions "absolues" immuables; nous noterons, pour fixer les idées, $2 L$ la longueur de l'écran, la valeur numérique de la constante $L$ étant déterminée par le nombre de pixels dont dispose la machine; de la sorte, tout point d'un axe horizontal tracé au centre de l'écran correspond à un nombre réel compris entre $-L$ et $L$. Sur cet écran fixe, l'ordinateur est amené à représenter, suivant les instructions de l'utilisateur, des intervalles de longueurs variables. Ainsi, lorsque l'implémentateur souhaite visualiser le comportement local du cercle autour de l'origine, il impose au logiciel une représentation graphique $^{2}$ sur des intervalles de plus en plus restreints, par exemple : $[-0,2 ; 0,2]$, $[-0,1 ; 0,1],[-0,01 ; 0,01], \ldots ;$ mais la machine fournit une image tracée sur tout l'écran, de sorte que les abscisses des points de cette image varient dans l'intervalle $[-L ; L]$ introduit ci-dessus ${ }^{3}$. Pour chacun des intervalles encodés dans la machine, un logiciel graphique effectue en fait une homothétie dont le centre est l'origine et dont le rapport vaut respectivement $5 L, 10 L, 100 L, \ldots$ Ainsi qu'en atteste la figure 1, il est dès lors demandé à l'ordinateur de représenter le graphe d'une même fonction (à savoir la fonction $x \mapsto \sqrt{2-(x-1)^{2}}-1$ provenant de l'équation du cercle) sur des intervalles de plus en plus petits, l'output fourni par l'ordinateur consiste alors en des courbes différentes, mais tracées sur une même zone de l'écran.

En d'autres termes, la machine effectue une transformation du plan qui multiplie toutes les longueurs par le rapport de l'homothétie correspondante; de façon plus imagée, elle se comporte comme un microscope dont l'oculaire serait pointé ici sur l'origine $O$.

Nous noterons $\mathcal{M}_{n}^{O}$ la transformation du plan numérique réalisée par cette homothétie de rapport égal à $n$ et nous appellerons ce nombre $n$ la puissance $d u$ microscope $\mathcal{M}_{n}^{O}$. D'un point de vue analytique, ce microscope agit comme une application de $\mathbb{R}^{2}$ vers $\mathbb{R}^{2}$ définie par :

$$
\mathcal{M}_{n}^{O}:(x ; y) \mapsto(X ; Y)=(n x ; n y)
$$

${ }^{2}$ Nous supposerons ici que l'utilisateur impose à l'ordinateur de travailler avec un repère orthonormé.

${ }^{3}$ Observons toutefois que l'écran apparaît comme gradué selon les valeurs comprises dans l'intervalle encodé et non pas selon celles comprises entre $-L$ et $L$. 
ce qui donne naissance aux égalités suivantes reliant les variables initiales $x, y$ aux nouvelles variables $X, Y$ :

$$
x=\frac{X}{n} \quad \text { et } \quad y=\frac{Y}{n} .
$$

Nous appellerons image d'une courbe $\mathcal{C}$ par le microscope $\mathcal{M}_{n}^{O}$ la courbe dessinée sur l'écran par l'ordinateur : elle sera notée simplement $\mathcal{C}_{n}$. Une équation de $\mathcal{C}_{n}$ peut être obtenue en partant de l'équation de $\mathcal{C}$ et en y remplaçant $x$ par $\frac{X}{n}$ et $y$ par $\frac{Y}{n}$; elle s'exprime donc en les nouvelles variables $X$ et $Y$ qui varient dans l'intervalle $[-L ; L]$. Par exemple, quand nous demandons à l'ordinateur de tracer le cercle $\mathcal{C}$ de l'exemple introductif sur l'intervalle $[-0,1 ; 0,1]$, nous voyons sur l'écran la courbe $\mathcal{C}_{10 L}$ dont une équation est donnée par

$$
\left(\frac{X}{10 L}-1\right)^{2}+\left(\frac{Y}{10 L}+1\right)^{2}=2
$$

Le schéma suivant modélise la démarche explicitée ci-dessus à propos de la courbe initiale $\mathcal{C}$ d'équation $(x-1)^{2}+(y+1)^{2}=2$

$$
\begin{aligned}
& \text { Restriction Application Image pour } X \in[-L ; L] \\
& \rightarrow \quad[-1 ; 1] \quad \rightarrow \quad \mathcal{M}_{L}^{O} \quad \rightarrow \quad \mathcal{C}_{L} \equiv\left(\frac{X}{L}-1\right)^{2}+\left(\frac{Y}{L}+1\right)^{2}=2 \\
& \rightarrow[-0,2 ; 0,2] \rightarrow \mathcal{M}_{5 L}^{O} \quad \rightarrow \quad \mathcal{C}_{5 L} \equiv\left(\frac{X}{5 L}-1\right)^{2}+\left(\frac{Y}{5 L}+1\right)^{2}=2 \\
& \mathcal{C} \rightarrow[-0,1 ; 0,1] \rightarrow \mathcal{M}_{10 L}^{O} \quad \rightarrow \quad \mathcal{C}_{10 L} \equiv\left(\frac{X}{10 L}-1\right)^{2}+\left(\frac{Y}{10 L}+1\right)^{2}=2 \\
& \rightarrow[-0,01 ; 0,01] \rightarrow \mathcal{M}_{100 L}^{O} \quad \rightarrow \quad \mathcal{C}_{100 L} \equiv\left(\frac{X}{100 L}-1\right)^{2}+\left(\frac{Y}{100 L}+1\right)^{2}=2 \\
& \rightarrow\left[-\frac{1}{n} ; \frac{1}{n}\right] \rightarrow \mathcal{M}_{n L}^{O} \quad \rightarrow \quad \mathcal{C}_{n L} \equiv\left(\frac{X}{n L}-1\right)^{2}+\left(\frac{Y}{n L}+1\right)^{2}=2
\end{aligned}
$$

Evidemment, on peut orienter un microscope de puissance $n$ vers un point $P(r ; s)$ autre que l'origine. On se ramène alors aisément au cas précédent par translation. Analytiquement, ce microscope agit comme une application, qui sera notée $\mathcal{M}_{n}^{P}$, définie par

$$
\mathcal{M}_{n}^{P}:(x ; y) \mapsto(X ; Y)=(n(x-r) ; n(y-s)) .
$$

On dispose alors des égalités suivantes :

$$
x=r+\frac{X}{n} \quad \text { et } \quad y=s+\frac{Y}{n} .
$$




$$
\text { "antibi" — 2005/2/16 - 20:14 - page } 325-\# 7
$$

La figure 2 illustre l'application de microscopes de plus en plus puissants dirigés vers le point $P(2 ; 0)$.

\section{Activités pédagogiques}

L'utilisation d'un microscope virtuel $\mathcal{M}_{n}^{P}$ fournit une situation où apparaît naturellement un changement de repère; c'est également une opportunité d'appliquer des transformations géométriques du plan. En passant des variables initiales $x, y$ aux nouvelles variables $X, Y$, on effectue d'abord une translation pour que le point $P$ apparaisse au centre de l'oculaire, puis on réalise une homothétie de rapport $n$ pour multiplier par le facteur $n$ toutes les longueurs.

L'opération finalement réalisée est donc la composée d'une translation et d'une homothétie. Les propriétés géométriques de telles opérations pourront de la sorte être concrètement illustrées.

Revenons à notre exemple introductif du cercle $\mathcal{C}$. Nous constatons que les images $\mathcal{C}_{n}$ fournies par des microscopes de puissance $n$ centrés sur l'origine $O$ ou sur le point $P(2 ; 0)$ apparaissent à l'écran comme étant des droites, tout du moins à partir de certaines valeurs de $n$; analytiquement, cela s'explique par le fait que, dans l'équation de $\mathcal{C}_{n}$, les termes indépendants de $X$ et de $Y$ s'annulent et que, après multiplication des deux membres de l'équation par $n$, les termes subsistant et contenant un facteur en $\frac{1}{n}$ deviennent "négligeables" dès que la puissance $n$ est suffisamment grande.

Remarque. Dans le microscope, l'image d'une courbe convexe (resp. concave) voit sa courbure diminuer au fur et à mesure que la puissance du microscope grandit; à partir d'une certaine puissance, la courbe représentée par l'ordinateur prend la forme d'une droite.

Les considérations précédentes nous ont amené intuitivement à la détermination de la tangente en un point d'une courbe. Nous allons formaliser de façon plus précise ce phénomène en nous référant notamment à la notion de limite d'une suite de courbes, cette notion ayant été introduite dans [2].

\section{Rappels sur la notion de limite d'une suite de courbes}

Pour la bonne compréhension de la suite du texte, rappelons quelques fondements sur les suites de courbes; cette théorie est approfondie dans [2]. 
Pour une suite de courbes planes $\left(\mathcal{C}_{n}\right)_{n \in \mathbb{N}}$, nous dirons qu'une courbe $\overline{\mathcal{C}}$ en est une limite lorsque "la limite des équations $\operatorname{des} \mathcal{C}_{n}$ est une équation de $\overline{\mathcal{C}}$ ". Précisons cette définition de manière plus formelle.

\subsection{Limites de graphes de fonctions}

Considérons des fonctions $f_{n}$ à une variable définies sur un intervalle $I$. Si, pour tout $x \in I$, on a

$$
\lim _{n \rightarrow+\infty} f_{n}(x)=f(x),
$$

on dira que le graphe $\overline{\mathcal{C}}$ de $f$ est la limite des graphes $\mathcal{C}_{n}$ des $f_{n}$.

Exemples.

- Considérons les droites $\mathcal{D}_{n}$ d'équation

$$
y=\left(1-\frac{1}{n}\right) x
$$

pour tout naturel $n$. Leur limite est la droite $\overline{\mathcal{D}}$ d'équation $y=x$, puisque

$$
\lim _{n \rightarrow+\infty}\left(1-\frac{1}{n}\right) x=x .
$$

- La suite composée des graphes des polynômes de Taylor

$$
y=\sum_{k=0}^{n} \frac{(-1)^{k}}{(2 k+1) !} x^{2 k+1}
$$

a pour limite le graphe de la fonction $y=\sin x$.

Remarque. La notion de limite d'une suite de graphes de fonctions est basée sur celle de convergence uniforme. Il est en effet possible de construire des fonctions $f_{n}$ qui ne convergent pas absolument, mais dont la suite des graphes possède une limite.

En guise d'exemple, considérons les fonctions $f_{n}$ telles que, pour tout $x \in[0 ; 1]$ et $n$ entier naturel, on a

$$
f_{n}(x)= \begin{cases}2 n x & \text { si } x \in\left[0 ; \frac{1}{2 n}\right] \\ 0 & \text { si } \left.x \in] \frac{1}{2 n} ; 1\right]\end{cases}
$$




$$
\text { "antibi" — 2005/2/16 - 20:14 — page } 327 \text { - \#9 }
$$

La suite des graphes $\mathcal{C}_{n}$ de ces fonctions possède une limite, puisque, pour tout $x \in[0 ; 1], \lim _{n \rightarrow+\infty} f_{n}(x)=0$; néanmoins, ces fonctions $f_{n}$ ne convergent pas absolument sur l'intervalle considéré.

Nous sommes en présence d'une "illusion d'optique" : en effet, alors que les courbes $\mathcal{C}_{n}$ tendent vers la portion $\overline{\mathcal{C}}$ de l'axe horizontal comprise entre les abscisses 0 et 1 , les points $P_{n}\left(\frac{\alpha}{2 n} ; \alpha\right)$, pour tout $\left.\alpha \in\right] 0 ; 1\left[\right.$, appartiennent à $\mathcal{C}_{n}$ et tendent vers le point $(0 ; \alpha)$ situé sur l'axe vertical mais non sur $\overline{\mathcal{C}}$. Un tel phénomène est impossible en cas de convergence uniforme ([2]).

\subsection{Tangente à une courbe comme limite de sécantes}

Cette théorie sur les limites de courbes permet de clarifier certains points d'analyse mathématique. En effet, la tangente en un point d'une courbe est quelquefois présentée comme étant une droite dont la position est la limite d'une sécante variable ([1], p. 100), sans que ces notions soient bien précisées.

Pour éclaircir ce point, admettons que la tangente au graphe $\mathcal{C}_{f}$ d'une fonction $f$ au point $A(a ; f(a))$ est, sous réserve de l'hypothèse de dérivabilité de $f$ en $a$, la droite $T$ qui passe par $A$ et de coefficient directeur $f^{\prime}(a)$ ([3], p. 54). On dispose alors de ce résultat ([2]) :

Proposition 4.1. Soit $f$ une fonction dérivable en a. La tangente $T$ au graphe $\mathcal{G}_{f}$ au point $A(a ; f(a))$ est la limite des sécantes passant par les points $A$ et $P_{n}\left(a+\frac{1}{n} ; f\left(a+\frac{1}{n}\right)\right)$.

\subsection{Limites de courbes définies implicitement}

On considère un ensemble $E$ de $\mathbb{R}^{2}$ sur lequel sont définies les fonctions $f_{n}$, pour tout $n \in \mathbb{N}$; on note $\mathcal{C}_{n}$ la courbe définie implicitement par

$$
f_{n}(x ; y)=0 .
$$

S'il existe une fonction $f$, non identiquement nulle sur $E$, telle que, pour tout $(x ; y) \in E$ :

$$
\lim _{n \rightarrow+\infty} f_{n}(x ; y)=f(x ; y)
$$

on dira que la limite des $\mathcal{C}_{n}$ est la courbe d'équation

$$
f(x ; y)=0 .
$$




$$
\text { "antibi" — 2005/2/16 — 20:14 — page 328 — \#10 }
$$

Exemple. Pour tout naturel $n$, on considère l'ellipse $\mathcal{C}_{n}$ définie par

$$
x^{2}+\left(1+\frac{1}{n}\right) y^{2}=1 .
$$

La suite des $\mathcal{C}_{n}$ possède pour limite le cercle centré à l'origine et de rayon 1.

Remarque. Une courbe limite peut être dégénérée ou réduite à l'ensemble vide. Ainsi, la suite des ellipses d'équation $x^{2}+\frac{y^{2}}{n^{2}}=1$ possède pour limite la conique définie par $x^{2}=1$ : celle-ci est dégénérée en deux droites verticales d'équation $x=1$ et $x=-1$. Par ailleurs, la suite des hyperboles d'équation $x^{2}-\frac{y^{2}}{n^{2}}+1=0$ possède pour limite la conique d'équation $x^{2}+1=0$ : dans $\mathbb{R}^{2}$, il s'agit de l'ensemble vide.

Une activité intéressante pourrait d'ailleurs consister à chercher des liens entre la nature des courbes $\mathcal{C}_{n}$ d'une suite et celle de leur limite $\overline{\mathcal{C}}$, même dans des cas simples. Par exemple, on pourrait se demander quand une suite d'ellipses possède pour limite un cercle, ou une droite, ...

\section{Rectification de courbes par des zooms successifs}

\subsection{Linéarisation de fonctions dérivables}

V. Henry [11] a mis au point une séquence didactique visant à présenter l'opération mathématique de linéarisation; il s'agit de remplacer localement une courbe par sa tangente puisque, comme l'écrivait Cournot déjà en 1841, celle-ci est la droite dont la courbe s'écarte, dans un voisinage immédiat du point de contact, moins que toute autre droite menée par le même point ([8], p. 50).

Intuitivement, on souhaite observer, sur la courbe, des points fort proches d'un même point; à cet effet, on peut utiliser (du moins par la pensée) des microscopes dirigés vers le point considéré et de puissance $n$, puis passer à la limite pour $n$ tendant vers $+\infty$.

Traitons d'abord le cas d'une fonction $f$ dérivable en un point $a$ et regardons le graphe $\mathcal{C}$ de $f$ dans l'oculaire des microscopes $\mathcal{M}_{n}^{P}$, pour $n$ entier et $P(a ; f(a))$. La courbe $\mathcal{C}_{n}$ obtenue est d'équation :

$$
Y=n\left(f(a)-f\left(a+\frac{X}{n}\right)\right) .
$$

Il en résulte que la suite des courbes $\mathcal{C}_{n}$ possède pour limite la droite d'équation

$$
Y=f^{\prime}(a) X
$$




$$
\text { "antibi" — 2005/2/16 — 20:14 — page 329 — \#11 }
$$

Ces considérations nous permettent de définir la tangente au graphe d'une fonction dérivable comme suit :

DÉfinition 5.1. Pour une fonction $f$ dérivable en un point a, notons $\mathcal{C}_{n}$ les images du graphe de $f$ par les microscopes $\mathcal{M}_{n}^{P}$ où $P$ est le point $(a ; f(a))$. La tangente au graphe de $f$ est la droite passant par $P$ et parallèle à la limite de la suite des courbes $\mathcal{C}_{n}$.

\section{Réflexions didactiques}

La notion de tangente est présentée de diverses façons lors de la formation mathématique traditionnelle ([5], [12]), avec des changements de point de vue et de conceptions, par exemple avec une approche locale ou globale, une vision géométrique ou plus analytique ([12]). Dans les cours d'analyse, elle est souvent présentée soit comme limite de sécantes, soit comme "rectification" d'une courbe, c'est-à-dire comme une approximation locale d'une courbe par une droite; la première présentation est régulièrement développée sans définir avec précision ce qu'est la limite de droites, et la seconde conception vient généralement bien après la première sans nécessairement être reliée à celle-ci ([5]).

De ce point de vue, notre méthode semble posséder un double avantage. Non seulement elle définit de façon rigoureuse la limite d'une suite de courbes, en particulier la limite de sécantes, mais elle unifie les deux points de vue : la tangente est aussi bien la limite de droites que la limite des courbes obtenues par "zooms" successifs. De plus, cette approche peut donner une idée concrète de ce qu'est une tangente, à savoir une droite "confondue" avec la courbe au voisinage du point considéré. La méthode est ainsi susceptible de lever une objection formulée notamment par M. Fréchet à propos de la présentation classique, parfois formelle, de la notion de tangente dans les cours de mathématiques élémentaires : l'auteur rappelle que la notion de tangente n'est pas non plus une pure création de l'esprit : ce sont des considérations d'ordre physique qui en ont amené l'introduction, par exemple le sol sur lequel se déplace une voiture est tangent aux roues de cette voiture; il constate encore que, pour viser à la précision et à la généralité, les mathématiciens ont substitué à la définition expérimentale une définition abstraite ([9], p. 5).

L'usage du microscope virtuel répond à la critique de Fréchet : en effet, si, par la pensée, on regarde une roue de voiture à l'aide d'un micro- 
scope virtuel, on peut imaginer que la roue épouse parfaitement la route au point de contact.

Enfin, signalons qu'en faisant appel à des microscopes virtuels dont la puissance grandit, ou peut réellement imaginer la façon dont la tangente se construit progressivement par des zooms successifs. A ce propos, rappelons un principe pédagogique fort ancien, déjà donné en 1879 par J. Petersen : on comprend bien mieux une figure et on se la rappelle bien plus facilement quand on l'a vue pendant la période de construction (cité dans [6], p. 3).

\subsection{Zoom sur le graphe d'une fonction non dérivable}

De la même manière que pour des fonctions dérivables, le recours à un microscope virtuel se révèle encore efficace dans le cas d'une fonction non dérivable, mais la limite obtenue lorsque la puissance du microscope virtuel grandit indéfiniment est alors, sous réserve d'existence, une droite verticale dans le cas d'une tangente verticale, ou une demi-droite verticale dans le cas d'un point de rebroussement, ou encore est composée de deux demi-droites dans le cas d'un point anguleux.

Contentons-nous d'illustrer cette affirmation par des exemples simples; ils concernent les cas, respectivement, d'un point anguleux, d'une tangente verticale et d'un point de rebroussement.

Exemples.

- Considérons tout d'abord la fonction définie, pour $x \geq 0$, par l'égalité

$$
f(x)=\sqrt{x(x-1)^{2}} .
$$

Pointons un microscope virtuel de puissance $n$ sur le point $P(1 ; 0)$. L'image du graphe de $f$ est caractérisée par l'égalité suivante :

$$
\frac{Y}{n}=\sqrt{\left(1+\frac{X}{n}\right) \frac{X^{2}}{n^{2}}}
$$

ce qui permet d'écrire, en imposant $Y \geq 0$,

$$
\frac{Y^{2}}{n^{2}}=\frac{X^{2}}{n^{2}}+\frac{X^{3}}{n^{3}} \quad \text { ou encore } \quad Y^{2}=X^{2}+\frac{X^{3}}{n} .
$$

Un passage à la limite, pour $n$ tendant vers l'infini, livre

$$
Y^{2}=X^{2} \text { c'est-à-dire } Y=-X \text { si } X \leq 0 \quad \text { ou } \quad Y=X \text { si } x \geq 0 .
$$




$$
\text { "antibi" — 2005/2/16 — 20:14 — page 331 — \#13 }
$$

On retrouve ainsi les deux demi-tangentes au point anguleux $P$, à savoir les demi-droites d'équation

$$
y=-x+1 \text { pour } x \leq 1 \text { et } y=x+1 \text { pour } x \geq 1 .
$$

- Comme autre exemple, traitons le cas de la fonction $f$ définie par

$$
f(x)=1+\sqrt[3]{x-1}
$$

Un microscope virtuel de puissance $n$ dirigé vers le point $P(1 ; 1)$ donne pour image du graphe de $f$ la courbe d'équation

$$
\frac{Y}{n}=\sqrt[3]{\frac{X}{n}} \text { équivalente à } \frac{Y^{3}}{n^{2}}=X .
$$

En faisant tendre $n$ vers l'infini, on obtient à la limite la droite verticale d'équation $X=0$, ce qui montre que $f$ n'est pas dérivable en l'abscisse 1 et que le graphe de $f$ possède au point $P$ une tangente verticale d'équation $x=1$.

- Traitons enfin la courbe $\mathcal{C}$ d'équation

$$
y=\sqrt[3]{x^{2}}
$$

et zoomons autour de l'origine $O(0 ; 0)$. Un microscope virtuel $\mathcal{M}_{n}^{O}$ donne pour image la courbe d'équation

$$
\frac{Y}{n}=\sqrt[3]{\frac{X^{2}}{n^{2}}} ;
$$

on a donc $Y \geq 0$ et

$$
\frac{Y^{3}}{n}=X^{2}
$$

En prenant la limite pour $n$ tendant vers l'infini, on obtient

$$
X=0, \text { avec } \quad Y \geq 0 .
$$

L'origine est un point de rebroussement : la partie positive de l'axe des ordonnées est en effet une demi-tangente verticale de $\mathcal{C}$ au point $O$.

\subsection{Zoom sur un point d'une conique}

Dans un repère cartésien, considérons une conique $\mathcal{C}$ d'équation écrite sous une forme générale, à savoir :

$$
A x^{2}+2 B x y+C y^{2}+2 D x+2 E y+F=0
$$


où les trois paramètres $A, B, C$ ne sont pas simultanément nuls.

Fixons notre attention sur un point $P(r ; s)$ quelconque de $\mathcal{C}$ et pointons sur $P$ le microscope virtuel $\mathcal{M}_{n}^{P}$. L'image $\mathcal{C}_{n}$ de $\mathcal{C}$ dans l'oculaire est d'équation :

$$
\begin{aligned}
A\left(r+\frac{X}{n}\right)^{2}+2 B\left(r+\frac{X}{n}\right)(s+ & \left.\frac{Y}{n}\right)+C\left(s+\frac{Y}{n}\right)^{2} \\
& +2 D\left(r+\frac{X}{n}\right)+2 E\left(s+\frac{Y}{n}\right)+F=0 .
\end{aligned}
$$

En tenant compte de l'appartenance de $P$ à $\mathcal{C}$, l'égalité ci-dessus peut encore s'écrire, après quelques calculs élémentaires :

$$
(A r+B s+D) X+(B r+C s+E) Y+\frac{A X^{2}}{n}+\frac{2 B X Y}{n}+\frac{C Y^{2}}{n}=0 .
$$

Si l'on n'a pas simultanément $A r+B s+D=0$ et $B r+C s+E=0$, la suite des $\mathcal{C}_{n}$ possède comme limite, quand $n$ tend vers $+\infty$, la droite d'équation

$$
(A r+B s+D) X+(B r+C s+E) Y=0
$$

la tangente à $\mathcal{C}$ au point $P$ est dès lors la droite d'équation, dans le repère cartésien initial :

$$
(A r+B s+D)(x-r)+(B r+C s+E)(y-s)=0 .
$$

Nous retrouvons ainsi une règle pratique selon laquelle une équation de cette tangente s'obtient en remplaçant dans l'équation (1) de la conique $x^{2}$ par $r x, 2 x y$ par $s x+r y, y^{2}$ par $s y, 2 x$ par $x+r$ et $2 y$ par $y+s$. Remarquons encore que cette tangente est horizontale (resp. verticale) lorsque $A r+B s+D=0$ (resp. $B r+C s+E=0)$.

Exemple. Une application intéressante de la méthode du microscope consiste en la recherche des tangentes de direction donnée. A titre d'exemple, recherchons les tangentes horizontales et verticales à l'ellipse d'équation

$$
4(x-y)^{2}+9(x+y)^{2}=36 .
$$

Il s'agit de trouver les coordonnées $r$ et $s$ des points de tangence; puisque ceux-ci sont sur la courbe, on doit avoir

$$
13 r^{2}+10 r s+13 s^{2}=36
$$

En pointant un microscope virtuel de puissance $n$ sur le point $P(r ; s)$, on obtient, en tenant compte de l'égalité (2), la courbe $\mathcal{C}_{n}$ d'équation

$$
(26 r+10 s) X+(10 r+26 s) Y+\frac{13}{n}\left(X^{2}+Y^{2}\right)=0 .
$$




$$
\text { "antibi" — 2005/2/16 — 20:14 — page 333 — \#15 }
$$

En faisant tendre $n$ vers l'infini, on obtient comme limite la droite d'équation

$$
(13 r+5 s) X+(5 r+13 s) Y=0
$$

Les tangentes horizontales (resp. verticales) s'obtiennent en annulant le coefficient de $X$ (resp. de $Y$ ) dans l'égalité (3), puis en exploitant (2) pour déterminer les valeurs possibles pour $r$ et $s$. Ainsi, l'ellipse possède deux tangentes horizontales, à savoir les droites définies par

$$
y=\frac{\sqrt{13}}{2} \quad \text { et } \quad y=-\frac{\sqrt{13}}{2}
$$

respectivement aux abscisses

$$
x=\frac{-5}{2 \sqrt{13}} \quad \text { et } \quad x=\frac{5}{2 \sqrt{13}}
$$

Il existe aussi deux asymptotes verticales qui peuvent être obtenues en échangeant les rôles des abscisses et des ordonnées.

Dans le cas où $A r+B s+D=B r+C s+E=0$, le point $P$ est un point double de la conique $\mathcal{C}$ qui est alors forcément dégénérée. Dans ce cas, la méthode exposée ci-dessus s'applique encore aisément.

Exemple. En guise d'illustration simple, considérons l'hyperbole dégénérée d'équation cartésienne

$$
x^{2}-y^{2}-2 x+2 y=0
$$

et le point $P(1 ; 1)$. L'image de cette courbe dans l'oculaire du microscope $\mathcal{M}_{n}^{P}$ est d'équation

$$
\frac{X^{2}}{n^{2}}-\frac{Y^{2}}{n^{2}}=0 \text { équivalente à }(x-y)(x+y-2)=0 .
$$

La conique est dégénérée en les deux droites d'équations $x=y$ et $x+y=2$ qui se rencontrent au point $P$.

\section{Réflexions didactiques}

Dans un souci de simplification, nous avons choisi de ne traiter que le cas des coniques, mais la même méthode s'applique aussi simplement à toutes les courbes algébriques. Elle permet de la sorte d'éviter un recours au théorème des fonctions implicites, de trouver les tangentes verticales 
et de traiter le cas des points singuliers; elle s'avère dès lors non seulement simple, mais encore générale et efficace dans le cas des courbes algébriques. Il en va de même pour les graphes de fonctions analytiques lorsque les développements de Taylor sont connus. Ces prolongements sont étudiés en détail dans [12].

Bien plus, cette méthode nous semble apporter du sens dans la recherche des tangentes à une courbe. En effet, nous pensons que l'opération de "zoom" peut être aisément imaginée et que cette façon de présenter la tangente pourrait favoriser l'acquisition du sens des idées introduites. Par ailleurs, cette méthode peut être facilement mise en oeuvre car elle est systématique et ne requiert aucun calcul compliqué, ce qui devrait également permettre aux apprenants d'acquérir du sens par la pratique.

Ces hypothèses a priori sont examinées de manière détaillée dans [12]; dans cette thèse sont notamment analysées les réactions des élèves (au niveau de la facilité, de la motivation, du sens, ... ) vis-à-vis de diverses approches de la notion de tangente.

Par ailleurs, cette méthode se révèle particulièrement bien adaptée pour une présentation non-standard de l'analyse : il suffit dans ce cas de faire appel à un microscope virtuel de puissance infiniment grande et de retenir la partie "visible", c'est-à-dire de manière plus technique la partie standard, de l'image donnée de la courbe considérée par le microscope virtuel; une telle approche est également développée en profondeur notamment dans [10] et [12] et [15].

\section{Références}

[1] A. Adam et F. Lousberg, Espace Math, 54, De Boeck Wesmael, Bruxelles, 1998.

[2] A. Antibi, J. Bair et V. Henry, Limites de courbes : théorie et applications en analyse, Mathématique et Pédagogie 147 (2004), 65-87.

[3] A. Antibi et R. Barra, Transmath Term ES, programme 2002, Nathan, Paris, 2002.

[4] J. Bair et V. Henry, de l'Analyse Classique à l'Analyse Non Standard, Les cahiers de la mathématique appliquée, Ferrer et Céfal 1 (2004), 51-74.

[5] C. Castella, Apprendre avec et contre ses connaissances antérieures, Recherches en Didactique des Mathématiques 15-1 (1995), 7-47.

[6] R. Cuppens, Avec Cabri-Géomètre II, jouez ... et faites de la géométrie, Brochure de l'A.P.M.E.P. 136 (2002).

[7] A. Deledicq et M. Diener, Leçons de calcul infinitésimal, A. Colin, Paris, 1989. 


$$
\text { "antibi" — 2005/2/16 — 20:14 — page 335 — \#17 }
$$

[8] A. Cournot, Traité élémentaire de la théorie des fonctions et du calcul infinitésimal, 1841, Oeuvres complètes, tome VII, J. Vrin, Paris, 1984.

[9] M. Fréchet, Les mathématiques et le concret, Presses universitaires de France, Paris, 1955.

[10] V. Henry, Les hyperréels en analyse, Mathématique et Pédagogie 141 (2003), 47-58.

[11] V. Henry, Nouvelle approche pour introduire la notion de tangente à une courbe, Mathématique et Pédagogie 145 (2004), 65-79.

[12] V. Henry, Quelques questions soulevées par l'enseignement de l'analyse non standard à des économistes, thèse de didactique des mathématiques, Université Paul Sabatier de Toulouse, 2004.

[13] H. J. Keisler, Elementary Calculus, Prindle, Weber \& Scmidt, Boston, 1976.

[14] B. Pascal, L'esprit de la Géométrie - De l'art de persuader, Bordas, Paris, 1986.

[15] A. Petry, A propos des tangentes à une courbe, une présentation non standard, Bul. Belgian Math. Soc., in : A tribute to Maurice Boffa, 2001, 155-166.

ANDRÉ ANTIBI

UNIVERSITÉ PAUL SABATIER

118 ROUTE DE NARBONNE

31062 TOULOUSE CEDEX 4

FRANCE

E-mail: antibi@cict.fr

JACQUES BAIR

UNIVERSITÉ DE LIÈGE

BOULEVARD DU RECTORAT 7, BÂT. B31

4000 LIĖGE (SART TILMAN)

BELGIQUE

E-mail: J.Bair@ulg.ac.be

VALÉRIE HENRY

UNIVERSITÉ DE LIÈGE ET UNIVERSITÉ PAUL SABATIER DE TOULOUSE

E-mail: V.Henry@ulg.ac.be

(Received June, 2004) 